\title{
Enzymatic modules of the SAGA chromatin-modifying complex play distinct roles in Drosophila gene expression and development
}

\author{
Xuanying Li, ${ }^{1,2}$ Christopher W. Seidel, ${ }^{1}$ Leanne T. Szerszen, ${ }^{1}$ Jeffrey J. Lange, ${ }^{1}$ Jerry L. Workman, ${ }^{1}$ \\ and Susan M. Abmayr ${ }^{1,2}$ \\ ${ }^{1}$ Stowers Institute for Medical Research, Kansas City, Missouri 64110, USA; ${ }^{2}$ Department of Anatomy and Cell Biology, University \\ of Kansas Medical Center, Kansas City, Kansas 66160, USA
}

The Spt-Ada-Gen5-acetyltransferase (SAGA) chromatin-modifying complex is a transcriptional coactivator that contains four different modules of subunits. The intact SAGA complex has been well characterized for its function in transcription regulation and development. However, little is known about the roles of individual modules within SAGA and whether they have any SAGA-independent functions. Here we demonstrate that the two enzymatic modules of Drosophila SAGA are differently required in oogenesis. Loss of the histone acetyltransferase (HAT) activity blocks oogenesis, while loss of the H2B deubiquitinase (DUB) activity does not. However, the DUB module regulates a subset of genes in early embryogenesis, and loss of the DUB subunits causes defects in embryogenesis. ChIP-seq (chromatin immunoprecipitation [ChIP] combined with high-throughput sequencing) analysis revealed that both the DUB and HAT modules bind most SAGA target genes even though many of these targets do not require the DUB module for expression. Furthermore, we found that the DUB module can bind to chromatin and regulate transcription independently of the HAT module. Our results suggest that the DUB module has functions within SAGA and independent functions.

[Keywords: SAGA complex; deubiquitinase module; embryogenesis; transcription]

Supplemental material is available for this article.

Received April 25, 2017; revised version accepted August 11, 2017.

Histone modifications, such as acetylation and ubiquitination, play a key role in facilitating a number of nuclear events, including transcriptional regulation. Acetylation of histones is largely associated with relaxing chromatin structures in order to support the entry of transcriptional machinery to the genomic loci for activation, while histone ubiquitination has been linked to both gene activation and repression (Brownell et al. 1996; Weake and Workman 2008). The histone-modifying enzymes that catalyze these post-translational modifications are often integrated into large complexes to facilitate their enzymatic activity, substrate specificity, and genomic localization.

SAGA (Spt-Ada-Gcn5-acetyltransferase) is a 2-MDa transcription coactivator protein complex that contains several distinct modules of subunits. These include a transcription activator interaction module, a TATA-binding protein $(\mathrm{TBP})$ interaction module, and two enzymatic modules: the histone acetyltransferase (HAT) module

Corresponding authors: jlw@stowers.org, sma@stowers.org

Article published online ahead of print. Article and publication date are online at http://www.genesdev.org/cgi/doi/10.1101/gad.300988.117. Freely available online through the Genes \& Development Open Access option. and the deubiquitinase (DUB) module. Given the role of SAGA in transcription regulation, it is not surprising that some SAGA subunits are essential for development, especially subunits of the enzymatic modules. Gen5 is the acetyltransferase subunit of SAGA and ADA (yeast) and ATAC (metazoans) complexes (Grant et al. 1997; Martinez et al. 1998, 2001; Ogryzko et al. 1998; Guelman et al. 2006; Demeny et al. 2007). Drosophila Gen5 is required for metamorphosis and oogenesis (Carre et al. 2005). In mice, GCN5-null embryos die during embryogenesis (Xu et al. 2000), and GCN5 HAT activity is critical for cranial neural tube closure (Bu et al. 2007). The SAGA-specific HAT module subunit Ada2b, which is required for the HAT activity, is also essential for Drosophila viability (Kusch et al. 2003; Muratoglu et al. 2003; Qi et al. 2004; Pankotai et al. 2005; Zsindely et al. 2009).

The DUB module subunits also control developmental processes. In Drosophila, the Non-stop (DUB) and Sgf11

(C) $2017 \mathrm{Li}$ et al. This article, published in Genes \& Development, is available under a Creative Commons License (Attribution-NonCommercial 4.0 International), as described at http://creativecommons.org/licenses/ by-nc/4.0/. 
function together in neural development (Weake et al. 2008). The mouse DUB USP22 is critical for the early embryogenesis (Lin et al. 2012) and has also been shown to play a role in cell differentiation and lineage specification (Kosinsky et al. 2015).

Several studies have investigated the structural features of SAGA and how SAGA subunits integrate into the whole complex. The HAT module subunits were lost from SAGA when purified from an ada2 $\Delta$ strain, suggesting that the Ada2 is essential to anchor the HAT module (Lee et al. 2011). Similarly, Sgf73 anchors the DUB module into SAGA (Lee et al. 2009). These results are confirmed by separate groups using electron microscopy (EM) analysis and cross-linking studies (Han et al. 2014; Setiaputra et al. 2015). Moreover, the $\mathrm{N}$ terminus of Sgf73 is necessary for DUB module activation and its recruitment to SAGA. (Kohler et al. 2008, 2010). Notably, the DUB module can disassociate from SAGA under some conditions and remain stable. In yeast, a functional DUB module can be separated from SAGA by the proteasome regulatory particle (Lim et al. 2013). In Drosophila, loss of the Sgf73 homolog Ataxin-7 leads to disassociation of an enzymatically active DUB module (Mohan et al. 2014). In humans, the DUB module is still stable in SPT20 knockdown cells. Thus, the integrity of the DUB module appears to be independent of SAGA, suggesting that it may be able to exist as a free form (Nagy et al. 2009).

In this study, we took advantage of genetic approaches in Drosophila to remove the maternal contribution and investigated the requirement for SAGA HAT and DUB modules in early development. We found that perturbation of the HAT module caused defects in oogenesis. In contrast, DUB activity was expendable during oogenesis, and at least some early zygotic genes were transcriptionally active. Nevertheless, it was essential for normal cellularization in these early embryos and their survival through embryogenesis. It was also essential for wildtype levels of transcription. We identified binding sites for several SAGA subunits genome-wide in early embryos and found coincident binding at most sites. Notably, the DUB module bound to chromatin with other SAGA subunits even when it was not required for transcription of the associated genes. More interestingly, we identified sites in which DUB module subunits bound chromatin independently of the core SAGA modules, where it regulated transcription.

\section{Results}

The HAT module is required for oogenesis, whereas the $D U B$ is expendable

Since the SAGA complex contains distinct functional modules, we sought to investigate whether all of the modules of SAGA are required coordinately or whether different modules play distinct roles during development. We focused on the two enzymatic modules, which contain the HAT activity and the DUB activity. We first evaluated whether both the HAT activity and the DUB activity were required at the earliest stages of development, during oo- genesis. We used the FLP/FRT/OvoD system /Chou and Perrimon 1996) to delete subunits of the DUB and HAT modules in the germline cells of the germline clone (GLC) females' ovaries. This analysis was carried out with null alleles of SAGA-specific subunits Ada2b, which is required in the HAT module for its activity (Kusch et al. 2003; Muratoglu et al. 2003; Qi et al. 2004); Ataxin-7, which anchors the DUB module to the remainder of SAGA; and the DUB subunit Non-stop (Supplemental Fig. S1).

We found that oogenesis is blocked in Ada2b GLC females, which did not lay eggs. This result is consistent with previous data that $A d a 2 b$ germ cells arrest at an early stage of oogenesis (Qi et al. 2004). To determine the stage at which Ada2b was required, we examined the morphology of dissected ovaries. Overall, Ada2 $b$ mutant egg chambers developed to stage 10, when nuclei began to exhibit degeneration (Fig. 1A), and apoptotic cell death was observed (Supplemental Fig. S2). Differential interference contrast images also revealed degeneration of the oocyte prior to maturity (Supplemental Fig. S3). In contrast with the $A d a 2 b$ mutants, oogenesis proceeded normally in both the Ataxin-7 and non-stop GLC females, suggesting that oogenesis is not dramatically affected by disrupting the DUB activity of SAGA. Moreover, nuclei in these developing oocytes were normal (Fig. 1A), and the females laid eggs. Whole dissected ovaries show the dramatic impact

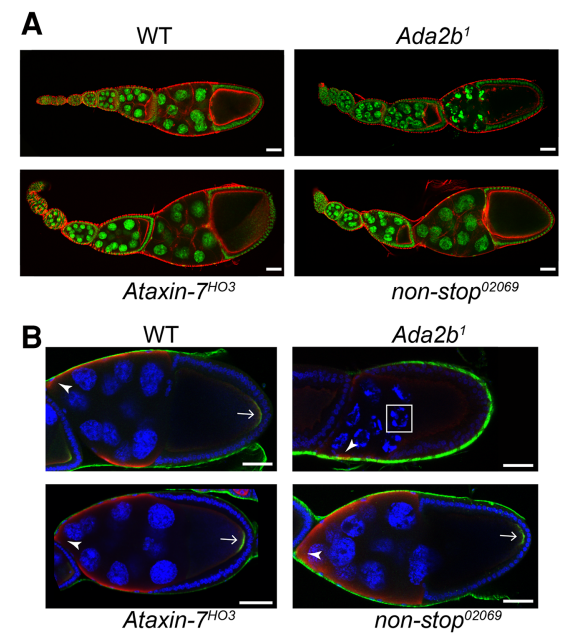

Figure 1. The HAT module is required for oogenesis, whereas the DUB is expendable. $(A)$ Whole ovaries were stained with phalloidin (red) for actin and DAPI (green) for nuclei. Posterior is to the right in all panels. $(B)$ Vasa protein was observed in the anterior end of stage 10 oocytes for all genotypes (red; arrowhead). Staufen was observed at the posterior end of wild-type, Ataxin- $7^{\mathrm{HO}}$, and non-stop 02069 GLC oocytes (green; arrow). The muscle sheath that surrounds the ovariole shows prominent but likely nonspecific staining with the Staufen antibody. DAPI (blue) revealed degenerating germline nurse cell nuclei in $A d a 2 b^{1}$ egg chambers (highlighted by a white box). The genotypes of GLC ovaries were as follows: (WT) Oregon R; $\left(A d a 2 b^{1}\right) h s-F l p /+; a d a 2 b^{1}$, $F R T^{82 B} / F R T^{82 B}$, ovo $^{D 1-18}$; (non-stop ${ }^{02069)}$ hs-Flp/+; nonstop ${ }^{02069}, F R T^{2 A} / F R T^{2 A}$, ovo ${ }^{D 1-18}$; (Ataxin-7 $\left.{ }^{H O 3}\right)$ hs-Flp/+; Ataxin-7 ${ }^{H O 3}$, FRT $^{40 A} / F^{\prime} T^{40 A}$, ovo ${ }^{D 1-18}$. Bars: $A, B, 50 \mu \mathrm{m}$. 
Li et al.

of loss of Ada2b and the normal state of the non-stop and Ataxin-7 GLCs (Supplemental Fig. S4). Egg laying rates for GLC females are shown in Supplemental Table S1.

To examine these mutant ovaries in more detail and begin to assess requirements for gene expression, we stained ovaries with the germline markers Vasa and Staufen, which play critical roles in germ plasm assembly during oogenesis (Brendza et al. 2000; Johnstone and Lasko 2001; Wilkie et al. 2003). Interestingly, we detected weak localized Vasa protein but no localized Staufen protein in the degenerating $A d a 2 b$ germline cells (Fig. 1B). However, Staufen was detected and localized to the posterior end of earlier stage oocytes (Supplemental Fig. S5). These results suggest that vasa and staufen mRNA transcription still occurs in the absence of Ada2b. Not surprisingly, in Ataxin-7 and non-stop GLC females' ovaries, those two proteins were still expressed and localized at the right places. Taken together, we conclude that the HAT module is required for normal oogenesis but not for transcription of all genes. However, oocytes lacking Non-stop or Ataxin-7 in the DUB module develop normally.

\section{The HAT module plays a critical role in transcriptional regulation in the ovary}

Although clearly some transcription occurred in ovary germ cells lacking Ada2b, as evidenced by the expression of Staufen and Vasa, the degeneration of these oocytes prompted us to investigate transcription defects genome-wide using RNA sequencing (RNA-seq). We hypothesized that comparison of gene expression in oocytes lacking Ada2b with those lacking Ataxin-7 or
Non-stop would allow us to identify genes requiring the SAGA HAT activity but not the DUB activity for their expression. The ovaries include both the germline-derived oocytes and overlying somatic follicle cells. The latter are wild type for these SAGA subunits in the GLCs. We were therefore unable to evaluate changes in expression of some genes in the oocytes if their expression in follicle cells was higher. However, this analysis did reveal those genes that differ between the HAT and the DUB modules in the ovary germline cells. Consistent with the severe phenotype, >1000 genes changed expression in $A d a 2 b$ GLC ovaries. In contrast, expression of only a few hundred genes was affected by absence of Ataxin-7 or Non-stop (Fig. 2A). We also found that very few genes overlapped between the three mutants, suggesting the possibility that the DUB module is exclusively required for the expression of some genes. Because of the presence of the overlying wild-type follicle cells, we are unable to identify genes that do not require the HAT module in the ovary. Nevertheless, these studies reveal different requirements for the DUB and the HAT modules in developing oocytes, with the latter playing a greater role.

To determine whether specific gene networks and pathways have a common requirement for the HAT activity during oogenesis, we performed gene ontology (GO) term analysis (Fig. 2B). Genes involved in DNA replication, eggshell formation, and chromosome organization were significantly down-regulated in the absence of Ada2b. Specifically, genes involved in DNA repair were down-regulated in Ada2b GLC ovaries, which may explain the nuclear degeneration phenotype (Supplemental Table S2). Similar analysis in Ataxin-7 or non-stop GLC ovaries revealed no significant enrichment. These data
A

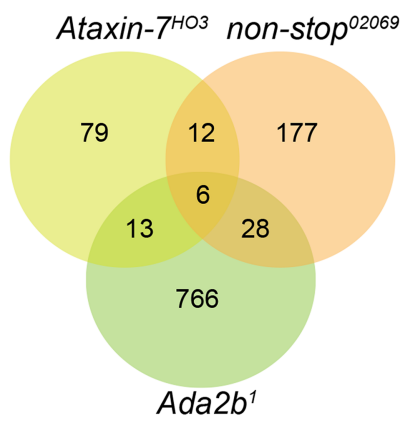

Down $>2$ fold, $\mathrm{P}<0.05$

B

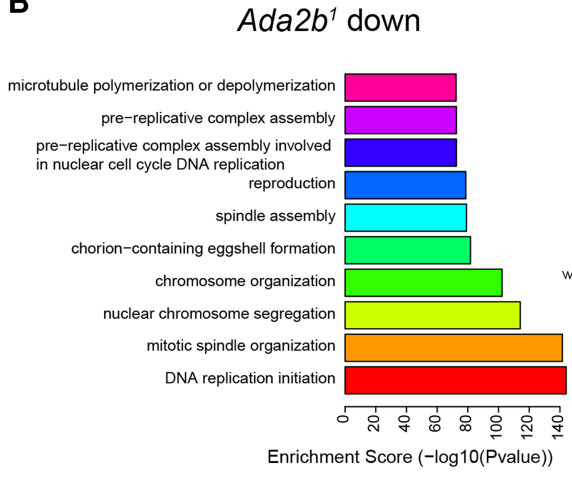

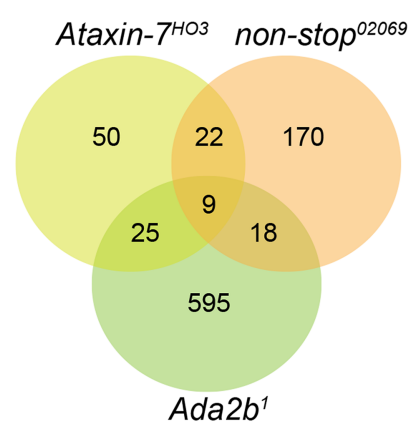

Up $>2$ fold, $\mathrm{P}<0.05$

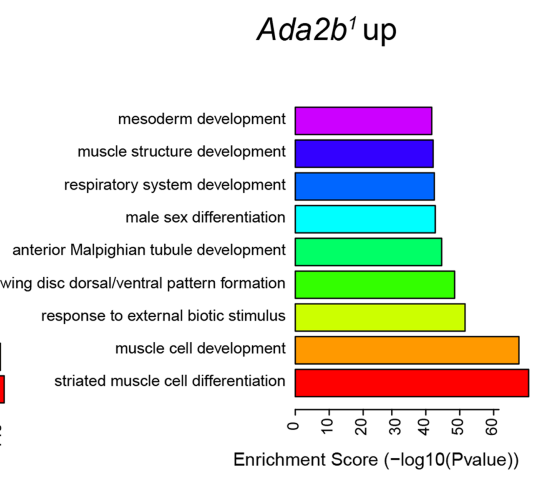

Figure 2. RNA-seq analysis reveals that the HAT module plays a critical role in transcriptional regulation in the ovary. $(A)$ Venn diagrams showing the overlapping genes with decreased or increased transcript levels in Ada $2 b^{1}$, Ataxin- $7^{H O 3}$, and non-stop 02069 GLC ovaries. Fold change $>2 ; P<0.05$; FPKM [fragments per kilobase per million mapped fragments] $>1$. $(B)$ Gene ontology $(\mathrm{GO})$ term analysis of the biological process (BP) of genes down-regulated or up-regulated in $A d a 2 b^{1}$ GLC ovaries. A complete list of enriched GO terms with an adjusted $P$-value of $<0.05$ is in Supplemental Table S2. 
correspond well with the different phenotypes shown earlier and support our earlier observations that nuclei degenerate in $A d a 2 b$ oocytes and that the DUB module is expendable for oogenesis.

\section{Early patterning still occurs in embryos lacking Ataxin-7 or non-stop}

To investigate whether the DUB module plays a role in embryogenesis, we examined the fertilized embryos from Ataxin-7 and non-stop GLC females. We focused on stage 5 embryos and confirmed from RNA-seq analysis that no maternal load or zygotic transcripts of Ataxin-7 or non-stop were yet detectable (Supplemental Fig. S1C). Therefore, we inferred that the presence of a wild-type zygotic copy from the male upon fertilization did not impact analysis at this early stage. We then examined the anterior-posterior $(\mathrm{A}-\mathrm{P})$ and dorsal-ventral (D-V) axes of these embryos. The pair rule gene even-skipped (eve) contributes to axis specification, and its zygotic expression is visible in a seven-stripe pattern along the A-P axis (Macdonald et al. 1986). The basic helix-loop-helix (bHLH) factor twist is one of the earliest zygotically active genes along the $\mathrm{D}-\mathrm{V}$ axis, and its expression along the ventral side of the embryos specifies mesoderm (Thisse et al. 1987). As shown in Figure 3A, Eve was apparent in a seven-stripe pattern in Ataxin-7 and non-stop GLC embryos in a pattern that is reminiscent of wild type, suggesting both that eve is expressed and that the A-P axis forms in the absence of DUB module subunits. We also found that twist was expressed in the presumptive mesoderm and localized on the ventral side, indicating formation of an early $\mathrm{D}-\mathrm{V}$ axis. We therefore conclude that transcription of some early zygotic genes occurs in early embryogenesis independent of the DUB module of SAGA.

\section{Loss of DUB module subunits affects cellularization and gene expression}

Although expression of some zygotic genes was initiated and early patterning occurred, we did observe defects in cellularization. Normally, the newly fertilized embryo undergoes several rounds of nuclear divisions without cytokinesis and remains syncytial. The nuclei then migrate to the periphery of the embryo, at which time they become surrounded by individual cell membranes (Mazumdar and Mazumdar 2002). In wild-type embryos, nuclei at the surface of the embryos formed an organized pattern (Fig. 3B, C). However, in Ataxin-7 and non-stop GLC embryos, nuclear Twist staining revealed a disorganized pattern, with the absence of nuclei in some locations. Expression of the membrane marker Discs-large (Dlg) revealed misshapen membranes in these regions. DAPI staining of all nuclei indicated that these defects were not limited to the ventral side (Fig. 4A). Notably, we were unable to distinguish the male chromosome provided to the egg upon fertilization by heterozygous mutant males and the genotype of the resulting embryos at this stage $\left(\mathrm{m}^{-/-}, \mathrm{z}^{-/-}\right.$vs. $\left.\mathrm{m}^{-/-}, \mathrm{z}^{-/+}\right)$. However, the above defects were observed in all embryos
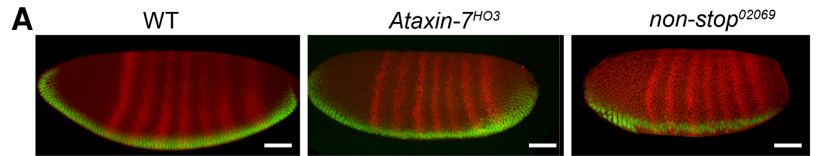

B

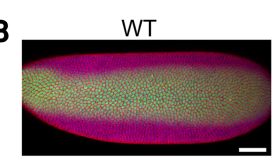

Ataxin- $7^{\text {HOз }}$
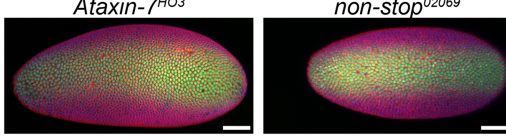

C
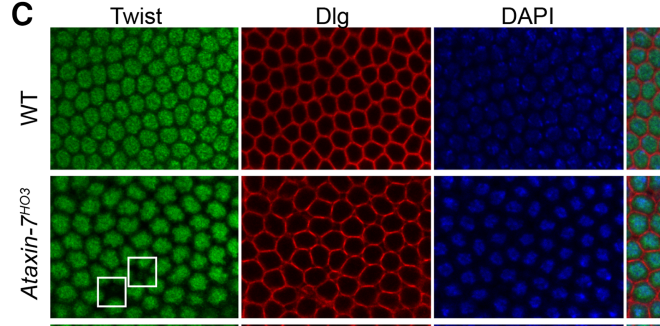

Merge
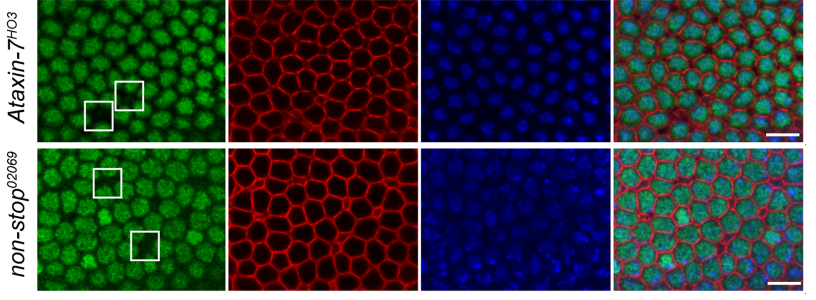

Figure 3. A-P patterning, segmentation, and mesoderm specification occur in Ataxin-7 and non-stop GLC embryos, but these embryo exhibit defects in cellularization. (A) Eve (red) staining was observed in the expected seven-stripe pattern in stage 5 GLC embryos, and Twist staining (green) revealed specified mesoderm on the ventral side of these embryos. Anterior is to the left and ventral is to the bottom. $(B)$ Stage 5 GLC embryos were stained with Discs-large (Dlg; red) to mark membranes, Twist (green) to mark mesodermal nuclei, and DAPI (blue) to mark all nuclei. A ventral view with anterior to the left is shown. Disruptions in the organization of Twist-expressing nuclei are apparent at this low magnification. $(C)$ High magnification of stage 5 embryos shows defects in organization of the migrated nuclei at the time of cellularization. Staining was as in B. Boxes denote positions in which nuclei on the surface appear to be absent. The embryos were collected from crosses as follows: (WT) Oregon R; (Ataxin-7 ${ }^{\mathrm{HO} 3}$ ) Ataxin-7 GLC females crossed to Ataxin-7 ${ }^{\mathrm{HO}}$ / Cyo; (non-stop ${ }^{02069}$ ) non-stop GLC females crossed to nonstop ${ }^{02069} /$ TM3. Bars: $A, B, 50 \mu \mathrm{m} ; C, 10 \mu \mathrm{m}$.

independent of the zygotic copy provided by the male. Last, the ovary and embryonic phenotypes of Ataxin$7^{\mathrm{HO} 3}$ were confirmed with Ataxin- $7^{2 A-1}$ (Supplemental Fig. S6). Thus, we conclude that these defects are a consequence of the absence of maternal Ataxin-7 or Non-stop.

Interestingly, we noted the presence of mislocalized nuclei in the interior of the embryo underneath the distorted regions (Fig. 4A). Time-lapse imaging revealed that these nuclei were actually able to migrate to the periphery but did not maintain this position and fell back into the interior of the embryos (Supplemental Movie S1). Despite the ability of most nuclei to remain at the periphery, these nuclei also exhibited defects in cellularization. Tubulin and Enabled revealed that cell membrane invagination was defective in GLC embryos (Fig. 4A). Real-time PCR showed that genes known to be involved in cellularization and nuclear anchoring (kuk, slam, bnk, Sry-a, and nullo) (Ibnsouda et al. 1993; Schejter and Wieschaus 1993; 


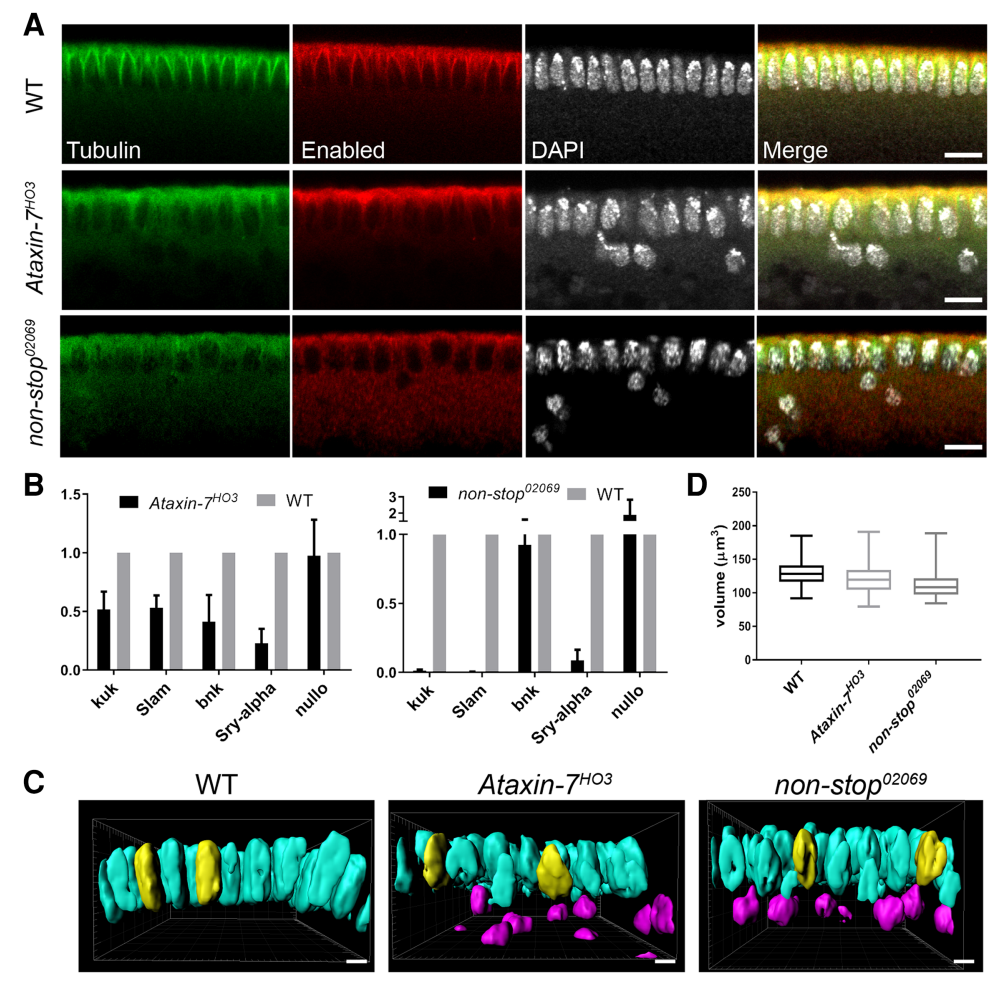

Figure 4. Similar defects in cellularization, gene expression, and nuclear shape are observed upon loss of the DUB module subunits Ataxin-7 and Non-stop. $(A)$ Stage 5 embryos were stained with a-Tubulin (green) and Enabled (red) to visualize invaginating membranes during cellularization. DAPI (gray) marked nuclei. $(B)$ Real-time PCR was performed on cDNA isolated from stage 5 wild-type, Ataxin- $7^{\mathrm{HO} 3}$, and non-stop ${ }^{02069}$ GLC embryos, and expression of genes associated with cellularization was assessed. Actin5C was used as normalization control. $(C)$ Screenshots of confocal data analyzed in three dimensions using Imaris to determine shape and volume for Twist-expressing nuclei. The embryo surface is at the top, and interior is at the bottom. Arbitrary coloring highlights nuclei at the surface (cyan), mislocalized nuclei (pink), and differences in nuclear shape (yellow). Three-dimensional movies are provided in Supplemental Movies S2-S4. (D) Quantification of nuclear volume of stage 5 embryos for each genotype, obtained from the Imaris analysis in $C$. Genotypes in $A-D$ were the same as in Figure 3. Bars: $A, 10 \mu \mathrm{m} ; C, 5$ $\mu \mathrm{m}$

Pritchard and Schubiger 1996; Stein et al. 2002; Brandt et al. 2006; Pilot et al. 2006; Chen et al. 2013) depend on the DUB module and change expression in non-stop and Ataxin-7 GLC embryos. In conjunction with similar loss-of-function phenotypes for kuk, non-stop, and Ataxin-7, we conclude that cellularization and nuclear anchoring are abnormal as a consequence of these decreases in expression. As shown in Figure 4B, transcript levels of these genes changed in the absence of Ataxin-7 or Non-stop (primer sequences are in Supplemental Table S3). Therefore, we conclude that the DUB module plays an important role in regulating transcription prior to cellularization.

We examined the muscle, central nervous system, $\mathrm{H} 3 \mathrm{~K} 9 \mathrm{ac}$, and H3K14ac in stage 16 Ada2b zygotic mutant embryos. The levels of H3K9ac and H3K14ac were reduced, indicating that the HAT module affects acetylation during embryogenesis, as shown previously (Qi et al. 2004). However, decreased acetylation at this stage does not result in phenotypic defects, possibly indicating that maternal Ada2b is sufficient for early transcription and specification (Supplemental Fig. S7A,B). We also identified Ada2b knockdown conditions that allowed oogenesis to proceed and examined the phenotype of the resulting embryos. The embryos exhibited defects in cellularization, indicating a role for Ada2b in early embryogenesis and suggesting that reduction in Ada2b has consequences similar to those of loss of Ataxin-7 and Non-stop (Supplemental Fig. S7C,D).

We also noted that most nuclei in Ataxin-7 and nonstop GLC embryos were misshapen. To better visualize these shapes and quantitate their volumes, we used the surfacing function of Imaris software. It revealed that a large number of nuclei at both the surface and interior did not adopt the elongated shape characteristic of wild type (Fig. 4C). The defective nuclei in GLC embryos appeared larger than wild type (Fig. 3C), but Imaris surfacing indicates that they are only modestly smaller (Fig. 4D). Given that all Ada2b GLC oocyte nuclei appeared to degenerate, we then asked whether these misshapen embryo nuclei in Ataxin-7 and non-stop GLCs were also degenerated. We examined the DNA damage marker $\gamma \mathrm{H} 2 \mathrm{Av}$ in Ataxin-7 GLC embryos, but this marker revealed no damaged nuclei (data not shown). Consistent with this finding, the early defects in embryos lacking the DUB module subunits could be rescued by zygotic expression of a wild-type copy. This rescue was apparent in the muscle pattern of Ataxin-7 $\left(\mathrm{m}^{-/-}, \mathrm{z}^{-/+}\right)$embryos despite their early defects (Supplemental Fig. S8A). In fact, $25 \%$ of these embryos survived to first instar larvae (Supplemental Fig. S8B). Thus, we conclude that the distorted shape and defective cellularization described above are not associated with degeneration of the nuclei and, moreover, that the embryo must be able to accommodate or correct these defects.

Despite the modest impact of the absence of DUB module subunits in early development, Ataxin-7 and Non-stop are important for embryogenesis. Notably, $75 \%$ of Ataxin-7 $\left(\mathrm{m}^{-/-}, \mathrm{z}^{-/+}\right)$embryos and $100 \%$ of nonstop $\left(\mathrm{m}^{-/-}, \mathrm{z}^{-/+}\right)$embryos died during embryogenesis (Supplemental Fig. S8B). More significantly, zygotic expression is essential, since embryos lacking both maternal and zygotic expression exhibited dramatic defects later in embryogenesis (Supplemental Fig. S8A), suggesting that the presence of the DUB module subunits is critical for embryogenesis. 
Altogether, these data demonstrate the importance of the DUB module for wild-type levels of gene expression in the early embryo and overt embryogenesis.

\section{The DUB module regulates a subset of genes in early embryogenesis}

Based on the known role of the DUB module in deubiquitination of $\mathrm{H} 2 \mathrm{~B}$ and the effect of its mutation on expression of several genes above, we wished to explore whether dependence on the DUB module at this stage extended beyond genes associated with cellularization. RNA-seq revealed expression of $\sim 6000$ genes at stage 5, as expected (RPKM [reads per kilobase per million mapped reads] $>1$ ), but only $40 \%$ of those genes changed expression in Ataxin-7 and non-stop GLC embryos (Fig. 5A). These data support our phenotypic results that while defects were observed, initial patterning of the early embryos was relatively normal.

The similar cellularization defects in Ataxin-7 and nonstop GLC embryos shown above suggests that these proteins function together within the DUB module to regulate transcription. To address this hypothesis, we compared the patterns of gene expression in these GLC embryos and found that $~ 50 \%$ of those genes that changed
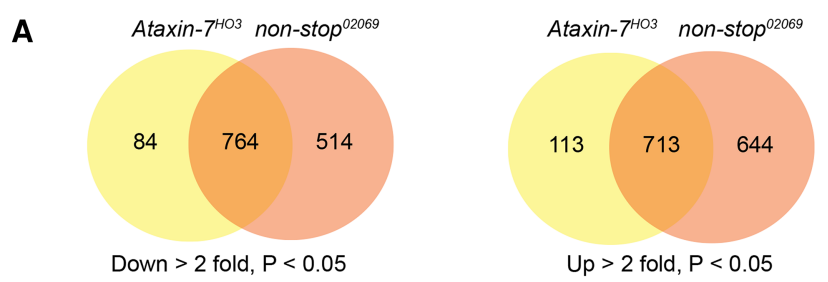

B

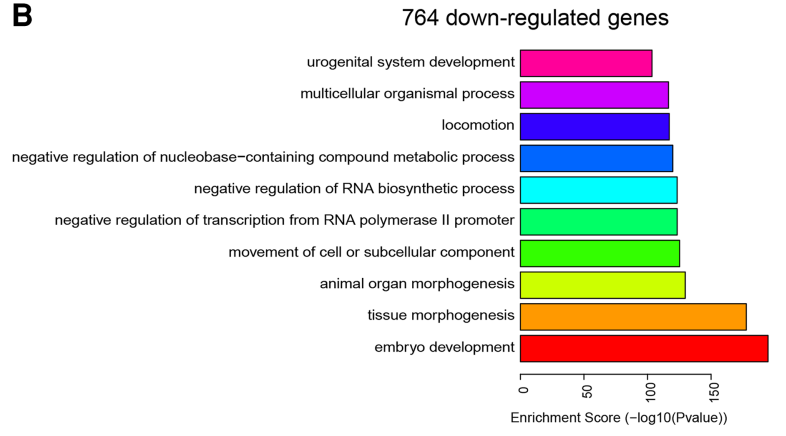

713 up-regulated genes

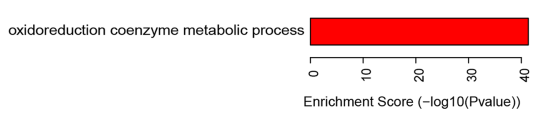

Figure 5. RNA-seq analysis reveals that the DUB module regulates expression of a subset of early genes in embryogenesis. (A) Venn diagrams showing the overlapping genes with decreased or increased transcript levels in stage 5 Ataxin- $7^{\mathrm{HO}}$ and nonstop $^{02069}$ GLC embryos. Fold change $>2 ; P<0.05$; FPKM $>1$. A large portion of genes was similarly affected in both mutants. $(B)$ GO term analysis of biological process $(\mathrm{BP})$ of genes commonly changed in both GLC embryos. A complete list of enriched GO terms with an adjusted $P$-value of $<0.05$ is in Supplemental Table S4. expression are similarly affected in both mutants (Fig. 5A; Supplemental Fig. S9). We then performed GO term analysis of the genes that are commonly changed. As shown in Figure 5B and Supplemental Table S4, down-regulated genes were enriched in several pathways, primarily those involved in embryonic development and tissue morphogenesis, indicating that these processes were highly dependent on the DUB module. Only genes associated with metabolic processes were enriched in the commonly up-regulated genes. As suggested by the GO term analysis (Supplemental Table S5), some of the up-regulated genes may be secondary effects of down-regulated repressors in the DUB mutants. Combined with the results of our phenotypic analysis, these findings demonstrate that the DUB module of SAGA regulates a subset of genes during early embryogenesis.

Previous studies have suggested that loss of DUB module subunits does not impair HAT activity and the integrity of the rest of SAGA (Weake et al. 2008; Lee et al. 2011; Mohan et al. 2014). To examine the HAT activity in Ataxin-7 GLC embryos, we performed ChIP-seq (chromatin immunoprecipitation [ChIP] combined with highthroughput sequencing) of $\mathrm{H} 3 \mathrm{~K} 9 \mathrm{ac}$ and $\mathrm{H} 3 \mathrm{~K} 14 \mathrm{ac}$ and found no significant change in Ataxin-7 GLC embryos. Moreover, Ada2b-binding sites did not change in Ataxin7 GLC embryos (Supplemental Fig. S10). We also examined $\mathrm{H} 3 \mathrm{~K} 9 \mathrm{ac}$ and $\mathrm{H} 3 \mathrm{~K} 14 \mathrm{ac}$ in the germline cells of Ataxin-7 and non-stop GLC ovaries and observed levels similar to those seen in wild type (Supplemental Fig. S11).

\section{Identification of SAGA-bound genes in early embryos}

To establish which genes were direct targets of SAGA, ChIP-seq assays were performed to determine occupancy in wild-type embryos. We chose subunits from each of three different SAGA modules: Ada2b in the HAT module, Spt3 in the SPT module, and Sgf11 in the DUB module. Multiple biological replicates were carried out, and peaks were called only if present in at least two replicates. We identified 3033 Ada2b peaks, 2382 Spt3 peaks, and 5425 Sgf11 peaks. To identify sites that likely bound the intact SAGA, we compared the binding patterns of these three factors for overlap and found good congruence (Fig. 6A). Here, we refer to the 1650 common peaks as SAGA peaks. Next, we assigned these peaks to the nearest transcription start sites (TSSs) and identified 1998 genes in which SAGA bound within 500 base pairs (bp) of the TSSs. Note that the number of target genes is greater than the number of peaks due to the fact that binding can occur within $500 \mathrm{bp}$ of the TSSs of one, two, or, in rare cases, three genes. As shown in Figure 6B and Supplemental Figure S9C, only some of the differentially expressed genes were bound by SAGA. These indirect targets may be secondary effects of down-regulated transcriptional activators in DUB mutants (Supplemental Table S5). Of the SAGA-bound targets, some showed expression changes in the DUB mutant (differentially expressed in DUB mutants; referred to here as DUB DE), but, interestingly, many others did not change (Fig. 6B). Thus, the DUB module binds to SAGA targets as a part of SAGA 
A

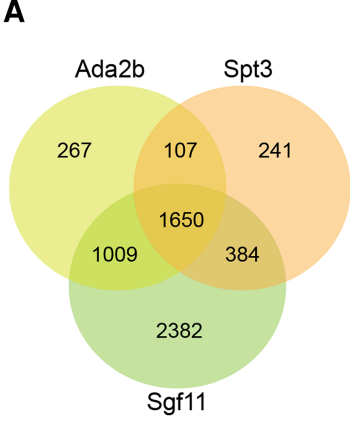

B

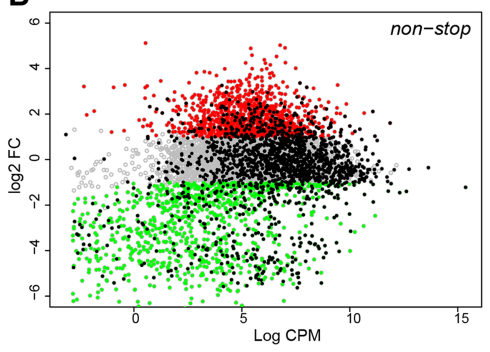

C
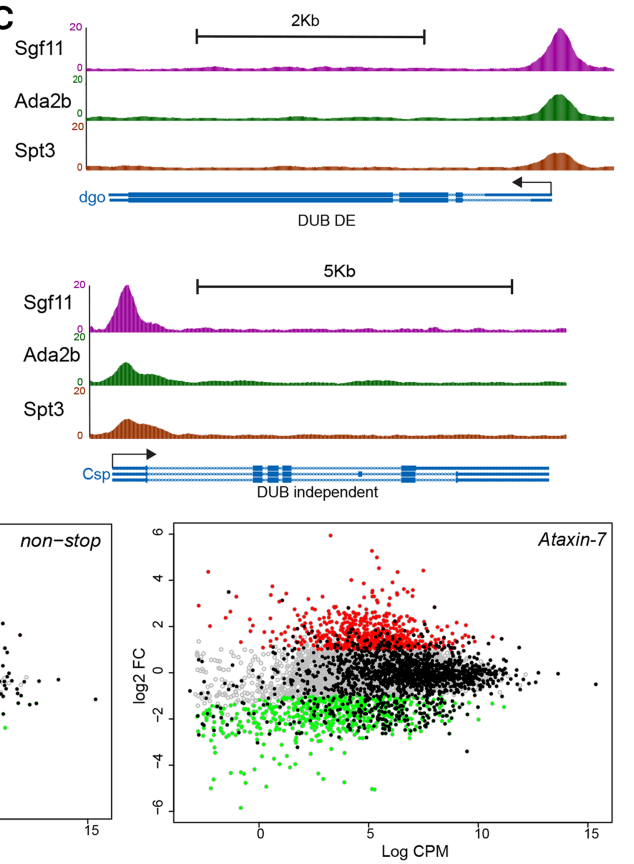

Figure 6. ChIP-seq analysis reveals that the DUB module binds to SAGA targets as a part of the whole complex but is required by only a subset of these genes for their expression. $(A)$ Venn diagram showing the overlapping ChIP-seq peaks between Sgf11, Ada2b, and Spt3. The overlapped peaks are referred to as SAGA peaks. $(B)$ MA plots showing gene expression in non-stop and Ataxin-7 GLC embryos (genes with FPKM $<1$ in wild type have been removed). Fold changes $>2$ are marked in red, and fold changes less than -2 are marked in green. Genes bound by SAGA are marked in black. $(C)$ Example genes show that the DUB module binds to SAGA targets even though it is not always required for their expression. Binding profiles for Sgf11 (purple), Ada2b (green), and Spt3 (orange) are shown at the $d g o$ (DUB DE gene) and Csp (DUB-independent gene) loci.

even though it is not always required for the expression of the target genes (Fig. 6C). We conclude that SAGA binds to many target genes as a whole complex in early embryos, but the DUB module is required by only a subset of these genes for their expression.

\section{The DUB module binds and regulates gene expression independent of the HAT module}

As noted above, some studies suggest that a stable DUB module exists independent of SAGA under some conditions. However, these studies did not address whether this module can bind to genomic chromatin independent of other SAGA modules under wild-type conditions. Whereas most of the Sgf11 peaks overlapped with Ada2b and Spt3, we noticed 2382 target sites where only Sgf11 was bound (Fig. 6A). We re-evaluated peaks using highly stringent criteria, including only those sites in which Ada2b and Spt3 binding was absent in all biological replicates. This analysis identified 647 Sgf11 peaks independent of Ada2b and Spt3 (Fig. 7A). MEME analysis showed that these sites include forkhead domain and homeobox domain motifs and are particularly enriched for zinc finger (Znf)-binding motifs (Supplemental Table S6). To address whether these Sgf11-bound sites reflected binding of the entire DUB module, we carried out ChIP analysis of Non-stop binding and found that $\sim 90 \%$ of all Sgf11 peaks were also bound by Non-stop (Supplemental Fig. S12). Since Non-stop was bound at $80 \%$ of the unique Sgf11 peaks, we inferred that these peaks represent SAGAindependent binding of an intact DUB module.

To examine the role of the DUB module in regulating transcription of novel targets, we assigned the 647 Sgf11-only peaks to the nearest TSS. Like SAGA, we found a similar binding preference of the DUB module near the
TSS, with 345 of these sites within $500 \mathrm{bp}$ of a TSS (Fig. 7B). Of the 345 DUB target genes (as identified by Sgf11 ChIP-seq), 56 genes required DUB subunits for their expression (Fig. 7C). Although the DUB module prefers to bind to TSS like SAGA does, it binds to genes with different types of promoters (Discussion; Supplemental Fig. S13A).

To examine the correlation of $\mathrm{ubH} 2 \mathrm{~B}$ and gene expression in the DUB mutants for the DUB targets and SAGA targets, we performed ChIP-seq of ubH2B in wild-type and Ataxin-7 GLCs. As shown in Supplemental Figure S14, down-regulated SAGA target genes have slightly decreased ubH2B in the coding regions in the absence of Ataxin-7, while up-regulated SAGA target genes have increased ubH2B in both promoters and coding regions (Supplemental Fig. S14B). In contrast, down-regulated DUB target genes showed decreased ubH2B in the coding regions in the absence of Ataxin-7, whereas up-regulated genes showed increased ubH2B in their promoters (Supplemental Fig. S14A). Thus, loss of Ataxin-7 leads to (1) increased ubH2B at up-regulated genes bound by either SAGA or DUB module subunits and (2) decreased ubH2B at down-regulated genes bound by either SAGA or DUB module subunits. This finding is consistent with an overall activating role of ubH2B. The different distribution of ubH2B in up-regulated genes in the DUB module targets (promoters) versus the SAGA targets (promoters and gene bodies) suggests that the DUB module plays distinct roles at those two sets of target genes.

We noticed throughout our analysis that a large portion of SAGA or DUB module target genes did not require the DUB module for their expression. We therefore wished to determine whether the SAGA target genes and the DUB target genes that did not require DUB for expression nevertheless had RNA polymerase II (Pol II) 
A
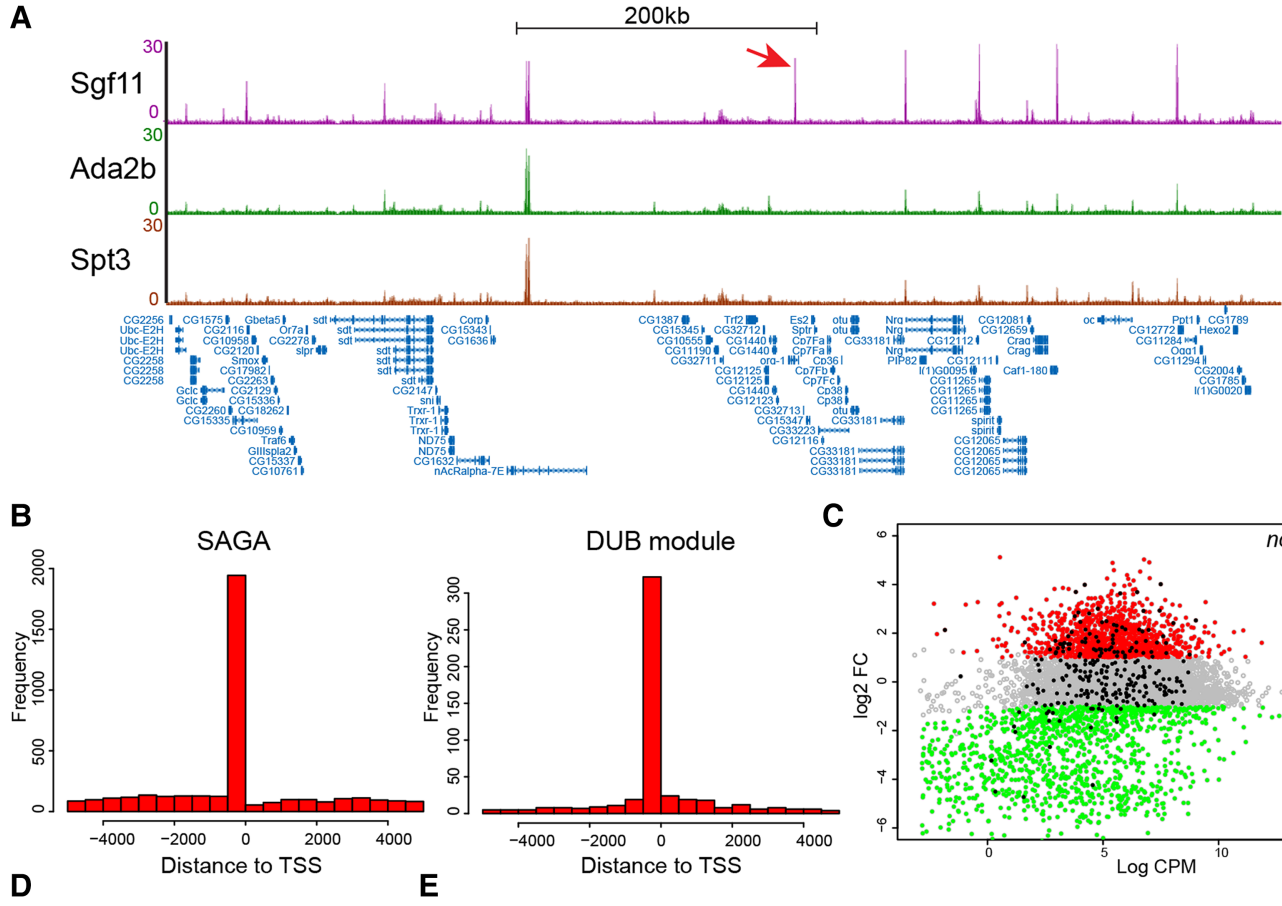

C
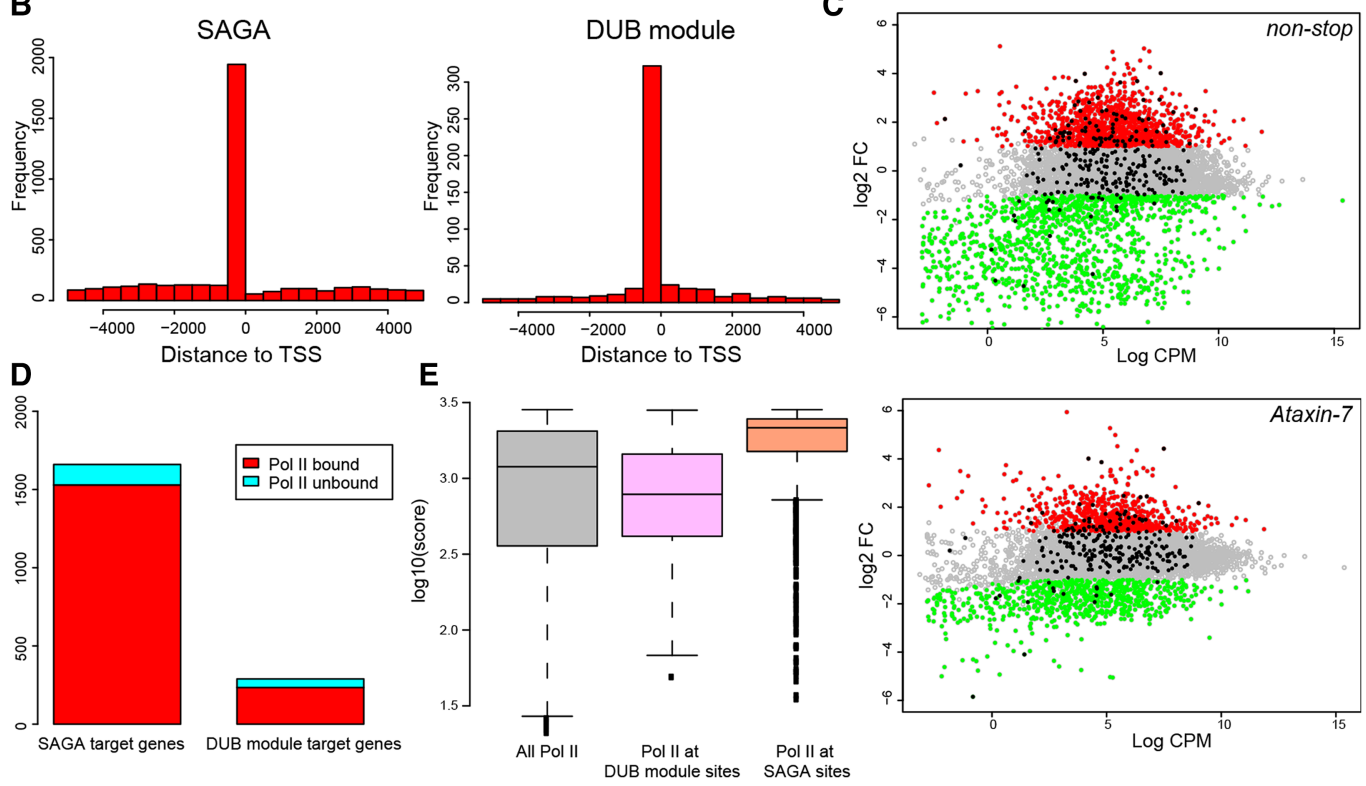

Figure 7. The DUB module binds and regulates gene expression independent of the HAT module. (A) Genome browser of ChIP-seq tracks at a representative region revealed that the DUB module binds to some genes without the HAT or SPT modules. The red arrow points to the Sgf11-only peak. $(B)$ Histograms of distance (from $-5000 \mathrm{bp}$ to $+5000 \mathrm{bp}$ ) to the TSSs for SAGA peaks and the DUB module peaks. $(C)$ MA plots showing gene expression in non-stop and Ataxin-7 GLC embryos (genes with FPKM $<1$ in wild type have been removed). Fold changes $>2$ are marked in red, and fold changes less than -2 are marked in green. Genes bound by the DUB module are marked in black. $(D)$ Bar plot showing that the SAGA target genes or the DUB module target genes that did not require the DUB module at this stage are bound by RNA polymerase II (Pol II). (E) To compare the Pol II levels at different sites, the $\log _{10}$ of Pol II peaks of expressed genes with an FPKM > 1 was plotted.

bound. The majority of these genes was indeed bound by Pol II (Fig. 7D). To further characterize the difference of the DUB DE and DUB-independent genes, we performed promoter analysis (Chen et al. 2013). As shown in Supplemental Figure S13A, for both SAGA targets and DUB targets, DUB DE genes and DUB-independent genes have distinct promoter types, indicating that different promoter types of genes have different requirements of the DUB module. DUB DE DUB targets are enriched for DPE and Inr promoter classes, while DUBindependent DUB targets are enriched for DRE and Ohler6. DUB DE SAGA targets are enriched for TATA, Inr, and MTE, while DUB-independent SAGA targets are enriched for DRE and Ohler1. Moreover, GO term analysis of SAGA targets suggested that dynamically expressed patterning genes are more likely to require the
DUB module, while metabolic process genes tend to be independent of the DUB module (Supplemental Fig. S15). Using intensity of $\mathrm{H} 2 \mathrm{~B}$ binding to monitor nucleosome occupancy between different classes of genes, we found no difference between DUB DE and DUB-independent targets for either DUB-specific or SAGA targets (Supplemental Fig. S13B).

Last, we examined the level of Pol II binding at expressed genes (Fig. 7E). Compared with all Pol II peaks of expressed genes (Fig. 7E, gray box), much higher levels of Pol II were present at SAGA-bound sites (Fig. 7E, orange box), consistent with previous findings (Weake et al. 2011). Pol II binding was not elevated at robustly expressed DUB target genes (Fig. 7E, pink box). However, the DUB module clearly facilitates Pol II binding genome-wide, possibly reflecting a role for the DUB module 
in the regulation of paused Pol II and elongation (Supplemental Fig. S16).

In summary, we conclude that the DUB module binds to chromatin as a part of SAGA and also independently of SAGA. Its presence is essential for transcription of some of these genes.

\section{Discussion}

The Drosophila HAT and DUB module subunits are required at different stages of development

Our results reveal the developmental stages at which the DUB and HAT modules of the SAGA chromatin-modifying complex are first required. Whereas the HAT module is required during oogenesis, the DUB module is not required for this process, and ovaries lacking Non-stop or Ataxin-7 are normal. Moreover, the $\mathrm{D}-\mathrm{V}$ and $\mathrm{A}-\mathrm{P}$ axes form in these embryos. The first defect observed in embryos lacking these DUB module subunits is at the stage of cellularization.

Numerous studies have explored the consequences of mutations in SAGA DUB and/or HAT module subunits at other stages of Drosophila and in other organisms. In some cases, loss of the two enzymatic modules has the same phenotypic impact, while in other cases, their loss has different consequences. In yeast, DUB module subunits Ubp8 and Sgf73 contribute to centromere stability, while the HAT module subunits Gen 5 and Ada3 do not (Canzonetta et al. 2015). An RNAi screen found that mouse GCN5 regulates reprogramming initiation in embryonic stem cells, whereas the DUB module appears to play no role in this process (Hirsch et al. 2015). In contrast, loss of the Drosophila Non-stop, Sgf11, and Ada2b causes similar defects in axon guidance in the eye imaginal disc (Weake et al. 2008). Loss of Gcn5 blocks oogenesis and metamorphosis (Carre et al. 2005), and zygotic Ada2b is also essential for viability (Qi et al. 2004; Pankotai et al. 2005). Although their mutant phenotypes have not been compared, the mouse USP22-, GCN5-, or ADA3-null embryos all die during embryogenesis (Xu et al. 2000; Lin et al. 2012; Mohibi et al. 2012). Additionally, GCN5 HAT activity is critical for cranial neural tube closure (Bu et al. 2007). Interestingly, loss of the Drosophila Ataxin-7 in the eye and adult brain results in neural degeneration (Mohan et al. 2014). Similarly, mammalian Ataxin-7 plays a primary role in the neurodegenerative disease spinocerebellar ataxia type 7 (David et al. 1997, 1998; Del-Favero et al. 1998; Johansson et al. 1998), and some genes that are regulated by Ataxin- 7 contribute to the pathology (Chen et al. 2012).

It is important to note in many of the above analyses that several SAGA subunits are present in multiple complexes. For example, Gcn 5 is also present in the ADA (yeast) and the ATAC (metazoans) complexes. Thus, the greater severity of Gcn5 mutants may result from the loss of these other complexes. Our studies with SAGAspecific subunits allow direct comparison of their requirement in regard to genetic phenotype, expression profiles, and target genes.
DUB and HAT module subunits control expression of a subset of genes

Many studies show that loss of SAGA subunits affects expression of a subset of genes and that different subunits seem to regulate distinct genes. In yeast, SAGA predominates at more regulated nonhousekeeping genes (de Jonge et al. 2017) and are preferentially used by those with TATA boxes, including those associated with stress (Basehoar et al. 2004; Huisinga and Pugh 2004). A more comprehensive genome-wide analysis of all viable SAGA deletion mutants suggests that different modules regulate different sets of genes (Helmlinger et al. 2011). Spt3 and Spt20 regulate $3 \%$ and $10 \%$ of yeast genes, respectively. Gen 5 has been reported to regulate between $1.1 \%$ and $4 \%$, most of which are distinct from those regulated by Spt3 or Spt20 (Lee et al. 2000; Helmlinger et al. 2008). Bonnet et al. (2014) suggested that SAGA is required for all transcribed genes based on Pol II recruitment and nascent mRNA synthesis at select genes. Gene expression profiling has also been reported for later stages in the Drosophila life cycle. Loss of zygotic Ada2b influences the expression of only $\sim 600$ genes in larvae and 900 genes in pupa (Zsindely et al. 2009; Pankotai et al. 2013; Pahi et al. 2015). Similar to yeast, many genes in Drosophila larvae require SAGA for their expression, but some are dependent on the DUB module, while others require the HAT module (Weake et al. 2008). Our results suggest that genes transcribed in the Drosophila ovary also have distinct requirements for the two enzymatic modules. Many genes require $\mathrm{Ada} 2 \mathrm{~b}$ in the ovary, while only a small number are affected by the loss of DUB module subunits.

Loss of these subunits alters transcription of many genes, while others exhibit robust expression that is unaffected. As noted above, we observed a reduction in expression of a subset of genes that are important for cellularization, which is likely responsible for the mutant phenotype. Our analysis showed that many genes are influenced by loss of only one of the subunits, but, consistent with their similar embryonic phenotypes, $\sim 50 \%$ of those genes impacted by the loss of Ataxin-7 or Nonstop are similarly affected in both mutants (Fig. 5A).

The possibility that other DUBs can substitute for Nonstop remains a formal possibility. There appear to be 23 candidate DUBs in Drosophila, several with significant homology with Non-stop. Scrawny and USP7 have been reported to have activity on $\mathrm{ubH} 2 \mathrm{~B}$ (van der Knaap et al. 2005; Buszczak et al. 2009). Scrawny encodes the Drosophila homology of yeast Ubp10, which has been reported to deubiquitinate $\mathrm{ubH} 2 \mathrm{~b}$ in the coding sequence (Schulze et al. 2011). It remains to be determined whether any of these can substitute in SAGA in the absence of Non-stop or whether another complex might provide this activity.

The DUB module binds to chromatin both as a part of the HAT-containing SAGA complex and independent of HAT module subunits

Previous genome-wide studies have used binding of a single SAGA subunit to infer targets of the entire complex. 
For example, Ada2b binding identified putative SAGA targets in Drosophila larva muscle and neurons (Weake et al. 2011), and SPT20 was shown to bind to a subset of transcribed genes in human cell lines (Krebs et al. 2011). In contrast to our analysis, these studies did not evaluate binding differences between modules.

We used three SAGA-specific subunits for three different modules in our whole-genome analysis of early embryos. Interestingly, we found that the DUB module subunits bound with HAT, and SPT module subunits bound to SAGA targets even though it was not always required for their expression. In addition, the DUB module bound to novel targets without the HAT or SPT modules in wild-type embryos. While we were unable to eliminate the possibility that the unique Sgf11 peaks reflect a robust antibody and are not actually devoid of HAT components, we note that different promoter types are enriched in SAGA targets and DUB-specific targets. This finding supports our view that the DUB targets are distinct from those of SAGA.

The mechanism by which the DUB module is recruited to chromatin is an interesting question. Sgf11 is a Znf protein and can bind to nucleosomal DNA (Koehler et al. 2014). Moreover, we found that Znf-binding sites are enriched in the Sgf11-specific targets (Supplemental Table S6). These findings support the hypothesis that DUB binds directly to DNA in the absence of the HAT and SPT modules via Sgf11. Sgf11-specific target sites are also enriched for forkhead domain and homeobox domain protein-binding motifs, supporting the possibility that this module binds to chromatin indirectly through nonSAGA proteins. Relevant to this issue, studies have already reported that the DUB module can interact with non-SAGA complexes. Under wild-type conditions in yeast, the proteasome chaperones interact with the Ubp8 and Sgf73 and separate them from SAGA (Lim et al. 2013). The yeast DUB module subunit Sus1 is also present in the mRNA export complex TREX-2 (Rodriguez-Navarro et al. 2004). The Drosophila Sus1 homolog $\mathrm{E}(\mathrm{y}) 2$ is also required for mRNA export (Kurshakova et al. 2007). These results suggest that the DUB module interacts with non-SAGA proteins and may have functions independent of SAGA. Further purification studies should address these various options and which proteins interact with the SAGA DUB module independent of the HAT module.

\section{Materials and methods}

Fly strains and culture

Drosophila melanogaster was grown on standard medium at $25^{\circ} \mathrm{C}$ unless stated otherwise. For GLC analyses, larvae were heatshocked for $2 \mathrm{~h}$ at $37^{\circ} \mathrm{C}$ for $2 \mathrm{~d}$ starting from the second day after hatching. Females were conditioned with yeast for 2-3 d before ovary dissection. Embryos were collected using standard apple juice plates with yeast. The non-stop ${ }^{02069}$ stock $\left(P\left\{r y^{+t 7.2}=P Z\right\}\right.$ not $\left.{ }^{02069} \mathrm{ry}^{506} / \mathrm{TM} 6 \mathrm{~B}, \mathrm{r}^{\mathrm{CB}} \mathrm{Tb}^{+}\right)$was provided by the Bloomington Drosophila Stock Center (BL11553) (Martin et al. 1995; Poeck et al. 2001). The $a d a 2 b^{1}$ fly stock was provided by Matthias Mannervik (Qi et al. 2004). The Ataxin- $7^{\mathrm{HO} 3}$ stock was generated by imprecise excision of the $\mathrm{P}$ element of Bloomington stock

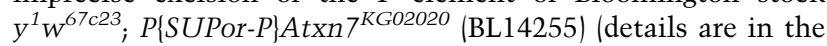
Supplemental Material)

\section{Immunostaining}

Dechorionated embryos were mixed vigorously in fixative $(2 \%$ formaldehyde, $0.1 \mathrm{M}$ PIPES at $\mathrm{pH} 6.9,2 \mathrm{mM} \mathrm{MgSO}_{4}, 1 \mathrm{mM}$ EDTA) with an equal volume of heptane for $18 \mathrm{~min}$ at room temperature.

Ovaries were dissected in Shields and Sang M3 insect medium and fixed in PBS with $4 \%$ formaldehyde for $15 \mathrm{~min}$ at room temperature.

Primary antibodies included Dlg (1:20), Eve (1:10), Enabled (1:500), and Fasciclin II (1:500) from Developmental Studies Hybridoma Bank; Twist (1:500); a-Tubulin (1:200; F2168) from Sigma-Aldrich; Vasa (1:200; sc-30210) and Staufen (1:200; sc15823) from Santa Cruz Biotechnology; and H3K9ac (ab4441) and H3K14ac (ab52946) from Abcam. Alexa fluor 647 phalloidin (1:20; Thermo Fisher Scientific, A22287) was used to detect F-actin. The myosin heavy chain monoclonal antibody (1:1000) was obtained from D. Kiehart. Alexa fluor secondary antibodies (1:200) were obtained from Invitrogen. Colorimetric detection used biotinylated anti-mouse secondary antibodies (1:200; Vector Laboratories) and the VectaStain ABC elite kit (Vector Laboratories). Cell nuclei were stained using $1 \mu \mathrm{g} / \mathrm{mL}$ DAPI.

Images were taken using Zeiss LSM 510 VIS and LSM 700 Falcon confocal microscopes. Some embryo and ovary images were generated by maximum projection of selected confocal slices using ImageJ. Channel colors were altered in Image for optimal viewing. Brightness and contrast were adjusted in ImageJ when necessary. Shape and volume were determined for Twist-stained nuclei using the surface function of Imaris.

\section{RNA purification and analysis}

Stage 5 embryos were collected and hand-sorted. Ovaries were dissected in Shields and Sang M3 insect medium. Total RNA was extracted using the RNeasy Plus minikit (Qiagen).

Real-time PCR was performed from biological triplicates using a 7900 HT real-time system. For RNA-seq analysis, biological triplicates were carried out for each genotype. Reads generated were 51-bp single-end poly-A-selected directional using the Illumina protocol. Resulting reads per gene were analyzed in $\mathrm{R}$ with the edgeR package using default methods. Differentially expressed genes were identified using the cutoff of $P<0.05$ and FPKM (fragments per kilobase per million mapped fragments $)>1$. The cutoff of fold change $>2$ was applied for identifying down-regulated genes and up-regulated genes.

\section{ChIP-seq analysis}

Stage 4-6 embryos were collected at $25^{\circ} \mathrm{C}$, and embryo stage was confirmed microscopically. Embryos were fixed in $1.8 \%$ formaldehyde for $15 \mathrm{~min}$ at room temperature (details are in the Supplemental Material). ChIP was performed as described previously (Huang et al. 2014).

The antibodies used for ChIP included $10 \mu$ g of rabbit $\alpha$-Sgf11, $20 \mu \mathrm{g}$ of rabbit $\alpha$-Ada $2 \mathrm{~b}, 20 \mu \mathrm{g}$ of rabbit $\alpha$-Spt $3,20 \mu \mathrm{g}$ of rabbit $\alpha$ Non-stop (details are in the Supplemental Material), $3 \mu \mathrm{g}$ of mouse a-Pol II (4H8) (Abcam, ab5408), $5 \mu \mathrm{g}$ of mouse a-ubH2B (EMD Millipore, 17-650), $5 \mu \mathrm{g}$ of rabbit $\alpha-\mathrm{H} 2 \mathrm{~B}$ (Abcam, ab1790), $5 \mu \mathrm{g}$ of rabbit $\alpha-\mathrm{H} 3 \mathrm{~K} 9 \mathrm{ac}$ (Abcam, ab4441), $5 \mathrm{\mu g}$ of rabbit $\alpha$-H3K14ac (Abcam, ab52946), and $5 \mu \mathrm{g}$ of rabbit $\alpha-\mathrm{H} 3$ (Abcam, ab1791). 
Reads of 51 bases from an Illumina HiSeq 2500 were aligned to version $\mathrm{dm} 3$ of the Drosophila genome from University of California at Santa Cruz using Bowtie using parameters -best -strata $-\mathrm{k} 1-\mathrm{m} 3$. The resulting BAM files were analyzed in R using Bioconductor to generate coverage and normalize the data in reads per million. Locations of enrichment for each protein were identified using MACS2 with default parameters.

ChIP-seq and RNA-seq data are available in Gene Expression Omnibus under accession number GSE98865.

\section{Acknowledgments}

Stocks obtained from the Bloomington Drosophila Stock Center (National Institutes of Health P40OD018537) were used in this study. We thank Developmental Studies Hybridoma Bank for maintaining some antibodies. We thank Dr. Ryan Mohan for collaborating with the Ataxin- $7^{\mathrm{HO} 3}$ stock. We thank Dr. Matthias Mannervik for providing the $a d a 2 b$ stock. We also thank Anoja Perera, Michael Peterson, Rhonda Egidy, Kate Hall, and Troy Green for running the RNA-seq and ChIP-seq samples. We thank William McDowell for assistance with real-time PCR. We thank Cynthia Chen and Hua Li for assistance with bioinformatics. We thank Steven Hoffman, Cindy Maddera, Jay Unruh, and Brian Slaughter for assistance with confocal images and imaging process. We thank all current and past Workman laboratory members for helpful comments and suggestions. This study was funded by the Stowers Institute and the National Institutes of General Medical Sciences (grants RO1GM099945 and R35GM118068) to S.M.A. and J.L.W. Original data are available at http://www. stowers.org/research/publications.

\section{References}

Basehoar AD, Zanton SJ, Pugh BF. 2004. Identification and distinct regulation of yeast TATA box-containing genes. Cell 116: 699-709.

Bonnet J, Wang CY, Baptista T, Vincent SD, Hsiao WC, Stierle M, Kao CF, Tora L, Devys D. 2014. The SAGA coactivator complex acts on the whole transcribed genome and is required for RNA polymerase II transcription. Genes Dev 28: 1999-2012.

Brandt A, Papagiannouli F, Wagner N, Wilsch-Brauninger $\mathrm{M}$, Braun M, Furlong EE, Loserth S, Wenzl C, Pilot F, Vogt N, et al. 2006. Developmental control of nuclear size and shape by Kugelkern and Kurzkern. Curr Biol 16: 543-552.

Brendza RP, Serbus LR, Duffy JB, Saxton WM. 2000. A function for kinesin I in the posterior transport of oskar mRNA and Staufen protein. Science 289: 2120-2122.

Brownell JE, Zhou J, Ranalli T, Kobayashi R, Edmondson DG, Roth SY, Allis CD. 1996. Tetrahymena histone acetyltransferase A: a homolog to yeast Gcn5p linking histone acetylation to gene activation. Cell 84: 843-851.

Bu P, Evrard YA, Lozano G, Dent SY. 2007. Loss of Gen5 acetyltransferase activity leads to neural tube closure defects and exencephaly in mouse embryos. Mol Cell Biol 27: 3405-3416.

Buszczak M, Paterno S, Spradling AC. 2009. Drosophila stem cells share a common requirement for the histone H2B ubiquitin protease scrawny. Science 323: 248-251.

Canzonetta C, Vernarecci S, Iuliani M, Marracino C, Belloni C, Ballario P, Filetici P. 2015. SAGA DUB-Ubp8 deubiquitylates centromeric histone variant Cse4. G3 6: 287-298.

Carre C, Szymczak D, Pidoux J, Antoniewski C. 2005. The histone $\mathrm{H} 3$ acetylase dGen 5 is a key player in Drosophila melanogaster metamorphosis. Mol Cell Biol 25: 8228-8238.
Chen YC, Gatchel JR, Lewis RW, Mao CA, Grant PA, Zoghbi HY, Dent SY. 2012. Gcn5 loss-of-function accelerates cerebellar and retinal degeneration in a SCA7 mouse model. Hum Mol Genet 21: 394-405.

Chen K, Johnston J, Shao W, Meier S, Staber C, Zeitlinger J. 2013. A global change in RNA polymerase II pausing during the Drosophila midblastula transition. Elife 2: e00861.

Chou TB, Perrimon N. 1996. The autosomal FLP-DFS technique for generating germline mosaics in Drosophila melanogaster. Genetics 144: 1673-1679.

David G, Abbas N, Stevanin G, Durr A, Yvert G, Cancel G, Weber C, Imbert G, Saudou F, Antoniou E, et al. 1997. Cloning of the SCA7 gene reveals a highly unstable CAG repeat expansion. Nat Genet 17: 65-70.

David G, Durr A, Stevanin G, Cancel G, Abbas N, Benomar A, Belal S, Lebre AS, Abada-Bendib M, Grid D, et al. 1998. Molecular and clinical correlations in autosomal dominant cerebellar ataxia with progressive macular dystrophy (SCA7). Hum Mol Genet 7: 165-170.

de Jonge WJ, O'Duibhir E, Lijnzaad P, van Leenen D, Groot Koerkamp MJ, Kemmeren P, Holstege FC. 2017. Molecular mechanisms that distinguish TFIID housekeeping from regulatable SAGA promoters. EMBO J 36: 274-290.

Del-Favero J, Krols L, Michalik A, Theuns J, Lofgren A, Goossens D, Wehnert A, Van den Bossche D, Van Zand K, Backhovens $\mathrm{H}$, et al. 1998. Molecular genetic analysis of autosomal dominant cerebellar ataxia with retinal degeneration (ADCA type II) caused by CAG triplet repeat expansion. Hum Mol Genet 7: 177-186.

Demeny MA, Soutoglou E, Nagy Z, Scheer E, Janoshazi A, Richardot M, Argentini M, Kessler P, Tora L. 2007. Identification of a small TAF complex and its role in the assembly of TAF-containing complexes. PLoS One 2: e316.

Grant PA, Duggan L, Cote J, Roberts SM, Brownell JE, Candau R, Ohba R, Owen-Hughes T, Allis CD, Winston F, et al. 1997. Yeast Gen 5 functions in two multisubunit complexes to acetylate nucleosomal histones: characterization of an Ada complex and the SAGA (Spt/Ada) complex. Genes Dev 11: $1640-1650$.

Guelman S, Suganuma T, Florens L, Swanson SK, Kiesecker CL, Kusch T, Anderson S, Yates JR III, Washburn MP, Abmayr SM, et al. 2006. Host cell factor and an uncharacterized SANT domain protein are stable components of ATAC, a novel dAda2A/dGcn5-containing histone acetyltransferase complex in Drosophila. Mol Cell Biol 26: 871-882.

Han Y, Luo J, Ranish J, Hahn S. 2014. Architecture of the Saccharomyces cerevisiae SAGA transcription coactivator complex. EMBO J 33: 2534-2546.

Helmlinger D, Marguerat S, Villen J, Gygi SP, Bahler J, Winston F. 2008. The pombe S SAGA complex controls the switch from proliferation to sexual differentiation through the opposing roles of its subunits Gen 5 and Spt8. Genes Dev 22: 3184-3195.

Helmlinger D, Marguerat S, Villen J, Swaney DL, Gygi SP, Bahler J, Winston F. 2011. Tral has specific regulatory roles, rather than global functions, within the SAGA co-activator complex. EMBO J 30: 2843-2852.

Hirsch CL, Coban Akdemir Z, Wang L, Jayakumaran G, Trcka D, Weiss A, Hernandez JJ, Pan Q, Han H, Xu X, et al. 2015. Myc and SAGA rewire an alternative splicing network during early somatic cell reprogramming. Genes Dev 29: 803-816.

Huang F, Paulson A, Dutta A, Venkatesh S, Smolle M, Abmayr SM, Workman JL. 2014. Histone acetyltransferase Enok regulates oocyte polarization by promoting expression of the actin nucleation factor spire. Genes Dev 28: 2750-2763. 
Huisinga KL, Pugh BF. 2004. A genome-wide housekeeping role for TFIID and a highly regulated stress-related role for SAGA in Saccharomyces cerevisiae. Mol Cell 13: 573-585.

Ibnsouda S, Schweisguth F, de Billy G, Vincent A. 1993. Relationship between expression of serendipity $a$ and cellularisation of the Drosophila embryo as revealed by interspecific transformation. Development 119: 471-483.

Johansson J, Forsgren L, Sandgren O, Brice A, Holmgren G, Holmberg M. 1998. Expanded CAG repeats in Swedish spinocerebellar ataxia type 7 (SCA7) patients: effect of CAG repeat length on the clinical manifestation. Hum Mol Genet 7: 171-176.

Johnstone O, Lasko P. 2001. Translational regulation and RNA localization in Drosophila oocytes and embryos. Annu Rev Genet 35: 365-406.

Koehler C, Bonnet J, Stierle M, Romier C, Devys D, Kieffer B. 2014. DNA binding by Sgf11 protein affects histone H2B deubiquitination by Spt-Ada-Gen5-acetyltransferase (SAGA). I Biol Chem 289: 8989-8999.

Kohler A, Schneider M, Cabal GG, Nehrbass U, Hurt E. 2008. Yeast Ataxin-7 links histone deubiquitination with gene gating and mRNA export. Nat Cell Biol 10: 707-715.

Kohler A, Zimmerman E, Schneider M, Hurt E, Zheng N. 2010. Structural basis for assembly and activation of the heterotetrameric SAGA histone H2B deubiquitinase module. Cell 141: 606-617.

Kosinsky RL, Wegwitz F, Hellbach N, Dobbelstein M, Mansouri A, Vogel T, Begus-Nahrmann Y, Johnsen SA. 2015. Usp22 deficiency impairs intestinal epithelial lineage specification in vivo. Oncotarget 6: 37906-37918.

Krebs AR, Karmodiya K, Lindahl-Allen M, Struhl K, Tora L. 2011. SAGA and ATAC histone acetyl transferase complexes regulate distinct sets of genes and ATAC defines a class of p300-independent enhancers. Mol Cell 44: 410-423.

Kurshakova MM, Krasnov AN, Kopytova DV, Shidlovskii YV, Nikolenko JV, Nabirochkina EN, Spehner D, Schultz P, Tora L, Georgieva SG. 2007. SAGA and a novel Drosophila export complex anchor efficient transcription and mRNA export to NPC. EMBO $J$ 26: 4956-4965.

Kusch T, Guelman S, Abmayr SM, Workman JL. 2003. Two Drosophila Ada2 homologues function in different multiprotein complexes. Mol Cell Biol 23: 3305-3319.

Lee TI, Causton HC, Holstege FC, Shen WC, Hannett N, Jennings EG, Winston F, Green MR, Young RA. 2000. Redundant roles for the TFIID and SAGA complexes in global transcription. Nature 405: 701-704.

Lee KK, Swanson SK, Florens L, Washburn MP, Workman JL. 2009. Yeast Sgf73/Ataxin-7 serves to anchor the deubiquitination module into both SAGA and Slik(SALSA) HAT complexes. Epigenetics Chromatin 2: 2.

Lee KK, Sardiu ME, Swanson SK, Gilmore JM, Torok M, Grant PA, Florens L, Workman JL, Washburn MP. 2011. Combinatorial depletion analysis to assemble the network architecture of the SAGA and ADA chromatin remodeling complexes. Mol Syst Biol 7: 503.

Lim S, Kwak J, Kim M, Lee D. 2013. Separation of a functional deubiquitylating module from the SAGA complex by the proteasome regulatory particle. Nat Commun 4: 2641.

Lin Z, Yang H, Kong Q, Li J, Lee SM, Gao B, Dong H, Wei J, Song J, Zhang DD, Fang D. 2012. USP22 antagonizes p53 transcriptional activation by deubiquitinating Sirt 1 to suppress cell apoptosis and is required for mouse embryonic development. Mol Cell 46: 484-494.
Macdonald PM, Ingham P, Struhl G. 1986. Isolation, structure, and expression of even-skipped: a second pair-rule gene of Drosophila containing a homeo box. Cell 47: 721-734.

Martin KA, Poeck B, Roth H, Ebens AJ, Ballard LC, Zipursky SL. 1995. Mutations disrupting neuronal connectivity in the Drosophila visual system. Neuron 14: 229-240.

Martinez E, Kundu TK, Fu J, Roeder RG. 1998. A human SPT3TAFII31-GCN5-L acetylase complex distinct from transcription factor IID. J Biol Chem 273: 23781-23785.

Martinez E, Palhan VB, Tjernberg A, Lymar ES, Gamper AM, Kundu TK, Chait BT, Roeder RG. 2001. Human STAGA complex is a chromatin-acetylating transcription coactivator that interacts with pre-mRNA splicing and DNA damage-binding factors in vivo. Mol Cell Biol 21: 6782-6795.

Mazumdar A, Mazumdar M. 2002. How one becomes many: blastoderm cellularization in Drosophila melanogaster. Bioessays 24: 1012-1022.

Mohan RD, Dialynas G, Weake VM, Liu J, Martin-Brown S, Florens L, Washburn MP, Workman JL, Abmayr SM. 2014. Loss of Drosophila Ataxin-7, a SAGA subunit, reduces H2B ubiquitination and leads to neural and retinal degeneration. Genes Dev 28: 259-272.

Mohibi S, Gurumurthy CB, Nag A, Wang J, Mirza S, Mian Y, Quinn M, Katafiasz B, Eudy J, Pandey S, et al. 2012. Mammalian alteration/deficiency in activation 3 (Ada3) is essential for embryonic development and cell cycle progression. I Biol Chem 287: 29442-29456.

Muratoglu S, Georgieva S, Papai G, Scheer E, Enunlu I, Komonyi O, Cserpan I, Lebedeva L, Nabirochkina E, Udvardy A, et al. 2003. Two different Drosophila ADA2 homologues are present in distinct GCN5 histone acetyltransferase-containing complexes. Mol Cell Biol 23: 306-321.

Nagy Z, Riss A, Romier C, le Guezennec X, Dongre AR, Orpinell M, Han J, Stunnenberg H, Tora L. 2009. The human SPT20containing SAGA complex plays a direct role in the regulation of endoplasmic reticulum stress-induced genes. Mol Cell Biol 29: 1649-1660.

Ogryzko VV, Kotani T, Zhang X, Schiltz RL, Howard T, Yang XJ, Howard BH, Qin J, Nakatani Y. 1998. Histone-like TAFs within the PCAF histone acetylase complex. Cell 94: 35-44.

Pahi Z, Kiss Z, Komonyi O, Borsos BN, Tora L, Boros IM, Pankotai T. 2015. dTAF10- and dTAF10b-containing complexes are required for ecdysone-driven larval-pupal morphogenesis in Drosophila melanogaster. PLoS One 10: e0142226.

Pankotai T, Komonyi O, Bodai L, Ujfaludi Z, Muratoglu S, Ciurciu A, Tora L, Szabad J, Boros I. 2005. The homologous Drosophila transcriptional adaptors ADA2a and ADA2b are both required for normal development but have different functions. Mol Cell Biol 25: 8215-8227.

Pankotai T, Zsindely N, Vamos EE, Komonyi O, Bodai L, Boros IM. 2013. Functional characterization and gene expression profiling of Drosophila melanogaster short dADA2b isoform-containing dSAGA complexes. BMC Genomics 14: 44.

Pilot F, Philippe JM, Lemmers C, Chauvin JP, Lecuit T. 2006. Developmental control of nuclear morphogenesis and anchoring by charleston, identified in a functional genomic screen of Drosophila cellularisation. Development 133: 711-723.

Poeck B, Fischer S, Gunning D, Zipursky SL, Salecker I. 2001. Glial cells mediate target layer selection of retinal axons in the developing visual system of Drosophila. Neuron 29: 99-113.

Pritchard DK, Schubiger G. 1996. Activation of transcription in Drosophila embryos is a gradual process mediated by the nucleocytoplasmic ratio. Genes Dev 10: 1131-1142. 
Li et al.

Qi D, Larsson J, Mannervik M. 2004. Drosophila Ada2b is required for viability and normal histone $\mathrm{H} 3$ acetylation. Mol Cell Biol 24: 8080-8089.

Rodriguez-Navarro S, Fischer T, Luo MJ, Antunez O, Brettschneider S, Lechner J, Perez-Ortin JE, Reed R, Hurt E. 2004. Sus1, a functional component of the SAGA histone acetylase complex and the nuclear pore-associated mRNA export machinery. Cell 116: 75-86.

Schejter ED, Wieschaus E. 1993. bottleneck acts as a regulator of the microfilament network governing cellularization of the Drosophila embryo. Cell 75: 373-385.

Schulze JM, Hentrich T, Nakanishi S, Gupta A, Emberly E, Shilatifard A, Kobor MS. 2011. Splitting the task: Ubp8 and Ubp10 deubiquitinate different cellular pools of H2BK123. Genes Dev 25: 2242-2247.

Setiaputra D, Ross JD, Lu S, Cheng DT, Dong MQ, Yip CK. 2015. Conformational flexibility and subunit arrangement of the modular yeast Spt-Ada-Gcn5 acetyltransferase complex. $I$ Biol Chem 290: 10057-10070.

Stein JA, Broihier HT, Moore LA, Lehmann R. 2002. Slow as molasses is required for polarized membrane growth and germ cell migration in Drosophila. Development 129: 3925-3934.

Thisse B, el Messal M, Perrin-Schmitt F. 1987. The twist gene: isolation of a Drosophila zygotic gene necessary for the establishment of dorsoventral pattern. Nucleic Acids Res 15: 3439-3453. van der Knaap JA, Kumar BR, Moshkin YM, Langenberg K, Krijgsveld J, Heck AJ, Karch F, Verrijzer CP. 2005. GMP synthetase stimulates histone $\mathrm{H} 2 \mathrm{~B}$ deubiquitylation by the epigenetic silencer USP7. Mol Cell 17: 695-707.

Weake VM, Workman JL. 2008. Histone ubiquitination: triggering gene activity. Mol Cell 29: 653-663.

Weake VM, Lee KK, Guelman S, Lin CH, Seidel C, Abmayr SM, Workman JL. 2008. SAGA-mediated H2B deubiquitination controls the development of neuronal connectivity in the Drosophila visual system. EMBO J 27: 394-405.

Weake VM, Dyer JO, Seidel C, Box A, Swanson SK, Peak A, Florens L, Washburn MP, Abmayr SM, Workman JL. 2011. Post-transcription initiation function of the ubiquitous SAGA complex in tissue-specific gene activation. Genes Dev 25: 1499-1509.

Wilkie GS, Dickson KS, Gray NK. 2003. Regulation of mRNA translation by $5^{\prime}$ - and $3^{\prime}$-UTR-binding factors. Trends Biochem Sci 28: 182-188.

Xu W, Edmondson DG, Evrard YA, Wakamiya M, Behringer RR, Roth SY. 2000. Loss of Gcn512 leads to increased apoptosis and mesodermal defects during mouse development. Nat Genet 26: 229-232.

Zsindely N, Pankotai T, Ujfaludi Z, Lakatos D, Komonyi O, Bodai L, Tora L, Boros IM. 2009. The loss of histone H3 lysine 9 acetylation due to dSAGA-specific dAda2b mutation influences the expression of only a small subset of genes. Nucleic Acids Res 37: 6665-6680. 


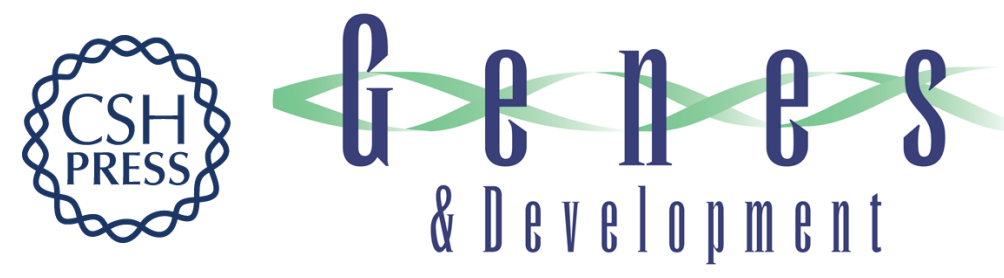

\section{Enzymatic modules of the SAGA chromatin-modifying complex play distinct roles in Drosophila gene expression and development}

Xuanying Li, Christopher W. Seidel, Leanne T. Szerszen, et al.

Genes Dev. 2017, 31: originally published online September 8, 2017

Access the most recent version at doi:10.1101/gad.300988.117

\section{Supplemental http://genesdev.cshlp.org/content/suppl/2017/09/08/gad.300988.117.DC1 \\ Material}

References This article cites 70 articles, 33 of which can be accessed free at:

http://genesdev.cshlp.org/content/31/15/1588.full.html\#ref-list-1

Creative This article, published in Genes \& Development, is available under a Creative Commons

Commons License (Attribution-NonCommercial 4.0 International), as described at

License http://creativecommons.org/licenses/by-nc/4.0/.

Email Alerting Receive free email alerts when new articles cite this article - sign up in the box at the top Service right corner of the article or click here.

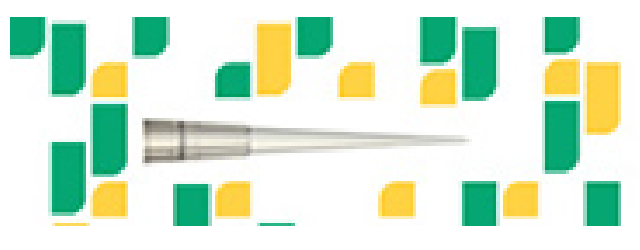

Focused on your science. 